\title{
Regulation of pre-osteoblast osteogenic transcription factors by different titanium surface topography
}

\begin{abstract}
The aim of the present study is to verify the morphogenic change and expression of Runx-2, Osterix, and osteocalcin $(\mathrm{OCN})$ of pre-osteoblasts on the titanium surfaces with machine polished process and MAO. The results indicate that the roughness, instead of the variant of methodology for metal surface conditioning, determines the response of MC3T3-E1 cells and the gene expression of Runx-2, Osterix, and OCN. The highest expression of Runx-2 and Osterix in smooth surface group happened late after 72 hours of incubation implied that the pre-osteoblasts showed a delay response of preosteogenesis on shining titanium surface compared to that of MAO group. The $1 \mu \mathrm{m}$ group showed the utmost expression of OCN as compared to those of smooth surface group and the two others groups. It can be concluded that roughness of $1 \mu \mathrm{m}$ may be the more preferred surface architecture for pre-osteoblasts to evolute and differentiate into osteoblasts, hence accelerate the process of osteogenesis.
\end{abstract}

Keywords: microarch oxidation, runx-2, osterix, osteocalcin, surface architecture
Volume 8 Issue 2 - 2017

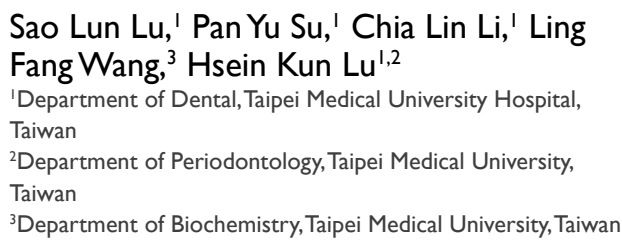

Correspondence: Hsein-Kun Lu, Department of Periodontology, College of Oral Medicine, Taipei Medical University, 250 Wu-Hsin Street, Taipei, Taiwan,

Email jackson@tmu.edu.tw

Received: August 3I, 2017 | Published: September 20, 2017

\section{Introduction}

Recent topic of discussion on dental implants revolves around differences in titanium surface topography. According to previous studies, different chemical or mechanical processing of titanium surface would elicit diverse cell or tissue reactions. Processes such as sandblasting, acid etching, or the even newer titanium oxide surface, render to excite more osteoblasts than the previous machined surface or plasma spray technique. Of all the parameters of surface processing, roughness and porosity are the two variables of relative importance. Macroscopically, the structure of processed surface increases the contact area per unit; and its bonding strength with bone tissue is strengthened from the mechanical undercut formed. Related animal study has revealed that once rabbit femur has achieved osteointegration with experimental implants, the torque removal would show obvious increase, achieving four times the value compared to the control implants with machined surface. ${ }^{1}$ Microscopically, the importance of surface architecture on the mechanism of osteointegration can be observed. When an implant is placed, hydration takes place between water molecules and titanium implant surface in a fraction of a second. At the same time, bonding occurs between calcium ion, phosphate ion and micromolecules, changing the electric charge on the titanium surface. Organic molecules such as amino acids, proteins, ester and peptide thus tightly attach to the hydrated interface, which is an important layer. ${ }^{2}$ Early in 1987 , Williams proposed that unique surface structure of implants can provide adherence for protein molecules. These protein molecules, when adhered to an implant surface, would form an organized structure to attract specific cells to bind and differentiate. ${ }^{3}$ In the process of bone formation and osteointegration, osteoblast differentiation is a key factor. ${ }^{4}$ Cell culturing and animal studies have discovered that different implant surface treatments would result in varied osteoblastic differentiation. ${ }^{5-9}$ Micro-arc oxidation (MAO) technique applies increasing voltage on metal compartments in an aqueous solution. As the oxidation rate increases on the metal, the surface structure recrystallizes, creating a local tunneling ionization reaction. Under the high temperature plasmatic effect, MAO-treated surface forms a layered structure. The outmost layer being porous and oxidized as the residue resulted from high temperature outburst of the treated metal. As the inner surface continues to be heated, it gradually acquires the properties of highly durable ceramics with high wear-resistance. With a dense oxidized layer and a outer porous structure to increase the route of ion diffusion, the metal of concern is more resistant to acid etching. ${ }^{10}$ In recent years, micro-arc oxidation technology has brought recognition in the surface oxidation of light metals such as aluminum, magnesium and titanium alloys, ${ }^{11}$ providing a simple and efficient way to process light metal surfaces. This porous structure significantly enhances the bonding of bone tissue and the titanium alloy surface. ${ }^{12}$ According to Boyan's study, culturing of osteoblasts on sandblasted and acid etched pure titanium surface would increase the formation of alkaline phosphatase (ALP) and type I collagen. ALP is a secreted enzyme in early stage of osteoblast differentiation. Type I collagen is the main protein secreted by osteoblasts, taking up to $90 \%$ of the bone matrix. Hence ALP and type I collagen are the two major detectors for osteoblast differentiation.

Runx-2 was extracted by Levanon et al in 1994. It is a transcription factor of the RUNX family. Other members of the RUNX family include Runx-1, which affects hemopoietic function, and Runx-3, which plays a role in the neural system. Runx-2 concerns the potential of osteo-differentiation. Inability to differentiate osteoblast and bone tissues that followed was observed on Runx 2 gene knockout mice. Once Runx-2 gene mutation occurs in human, autosomal dominant skeletal disorder and cleidocranial dysplasia would occur. Runx-2 is considered the key modulator in osteoblast differentiation, a major factor in achieving skeletal maturity and homeostasis. ${ }^{13}$ Osterix is a novel zinc finger-containing transcription factor, an important 
protein in osteoblast differentiation and bone formation. It is a direct downstream product of Runx 2 induced osteo-differentiation process. ${ }^{14}$ Under mechanical loading or the influence of vitamin $\mathrm{K}$ and vitamin D3, osteoblast would synthesize and excrete osteocalcin. Osteocalcin is the main non-collagen protein in bone cell matrix with size of 5800 daltons. Usually in the form of gamma-carboxy-glutamic acid (Gla), it binds to the minerals in the bone when calcium ion is present, and deposits in extracellular matrix. Being a transcription factor found solely in osteoblasts and no other extracellular matrixproducing cell, osteocalcin is an indicator of osteoblast activity and bone formation rate. ${ }^{15}$ Meanwhile, studies have shown that pore size on titanium surface can have a profound effect; the density of osteocytic differentiation on submicrometer surface is higher than micrometer surface. ${ }^{16}$ As nano technology progresses, interests in the effect of nano surface treatment on osteo-differentiation have spun. ${ }^{17}$ This study aims to investigate whether the pore size and roughness on metal of machined surface and MAO would alter the genetic expression of Runx-2 signaling pathway. By quantifying the results, an in vitro detecting method would be utilized to verify the ideal surface treatment to stimulate osteoblast differentiation and osteointegration, providing more information on surface treatment of dental implants.

\section{Material and method}

\section{Disk preparation}

Grade II commercially pure titanium was pre-fabricated into disks of $16 \mathrm{~mm}$ in diameter and $1.0 \mathrm{~mm}$ in thickness. With machined surface as control (Figure 1), surfaces were created by cathodically polarization at a constant current for 10 minutes in $1 \mathrm{M} \mathrm{H} 2 \mathrm{SO} 4$ solution at $298 \mathrm{~K}$, with a platinum plate used as a counter electrode. Various pore sizes were created with $10 \mu \mathrm{m}$ in roughness as group A (Figure 2), $1 \mu \mathrm{m}$ as group B (Figure 3), $0.1 \mu \mathrm{m}$ as group C (Figure 4) and $0.01 \mu \mathrm{m}$ as group D (Figure 5). Prior to experiment, deionized water and $5 \%$ sodium bicarbonate solution were used to cleanse the disks, further treated with ultrasonic shaker containing deionized water for three times of five minutes each. Autoclave was utilized to complete the sterilization. ${ }^{18}$

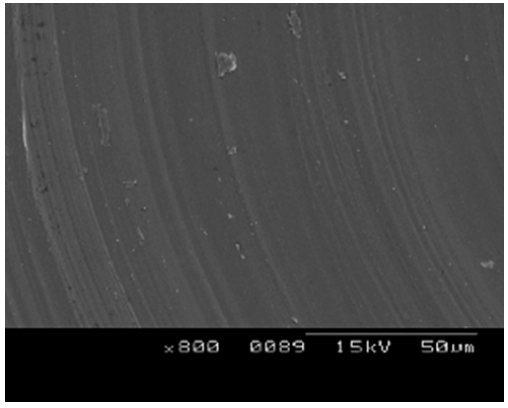

Figure I Machined surface under SEM.

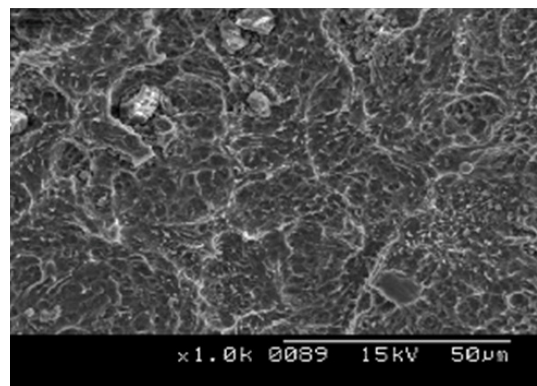

Figure $210 \mu \mathrm{m}$ surface under SEM.

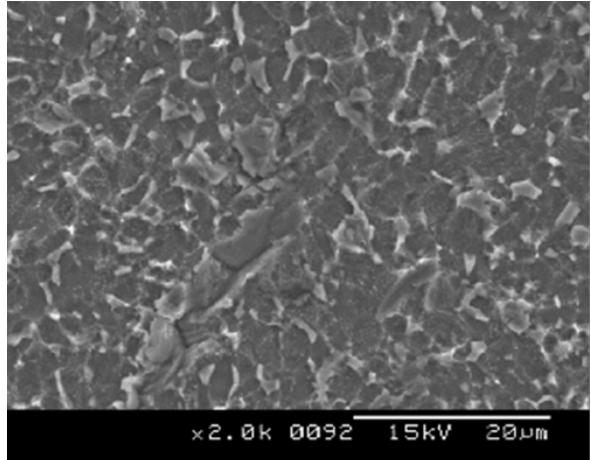

Figure $3 \mathrm{I} \mu \mathrm{m}$ surface under SEM

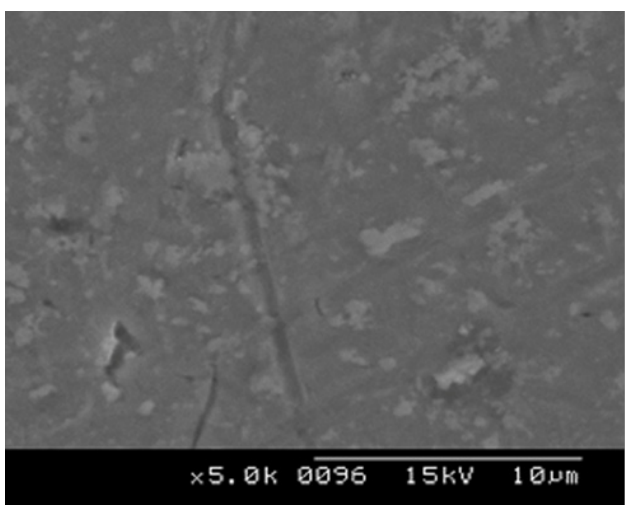

Figure $40.1 \mu \mathrm{m}$ surface under SEM.

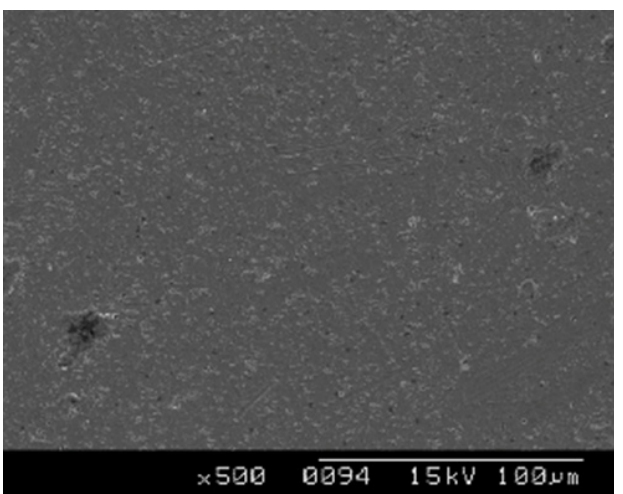

Figure 5 0.0I $\mu \mathrm{m}$ surface under SEM.

\section{Cell cultures}

MC3T3-E1 cell was used in this experiment. Cells were adhering to the titanium disks with high density culture technique. The cells were centrifuged to $2 \times 10^{4} / 500 \mu 1$ and transferred to the titanium disks placed in 16 well dishes, and placed in incubator $\left(5 \% \mathrm{CO}_{2}, 37^{\circ} \mathrm{C}\right)$ for one hour. After the cells had adhered, $\alpha$-MEM and $50 \mathrm{mg} / \mathrm{ml}$ ascorbate were added, and continue to incubate for 72 hours for quantifying examination.

\section{Scanning electron microscope (SEM)}

After 72 hour culture, $2.5 \%$ glutaraldehyde/paraformaldehyde fixative was used for MC3T3-E1 cell fixation. Dehydration was done with ascending series of alcohol, and critical point dried. Au/Pd layer was coated and the disks were examined under SEM with 10-20 kV beam voltage. 


\section{Real time-Q PCR}

Utilizing RT-Q PCR technique, Runx-2, osterix and osteocalcin gene expression of osteoblasts were investigated. After 72 hours of culturing, RNA was extracted using RNeasy Mini Kit. Firstly, spectrum analysis $(260 \mathrm{~nm})$ was performed to determine the concentration of RNA. Absorptivity was analyzed with 260 and $280 \mathrm{~nm}$. Module was made using the extracted RNA for reverse transcription with SYBR Green Reverse Transcription Reagents. Primer sets used were listed in Table 1.

Table I Primer sets used in RT-qPCR

\begin{tabular}{lll}
\hline \multirow{2}{*}{ Genes } & Primers sets & \\
\cline { 2 - 3 } & Forward & Reverse \\
\hline GAPDH & TGGAGAAACCTGCCAAGTATGGT & GTTGAAGTCACAGGAGACAAC \\
Runx-2 & AAGTGCGGTGCAAACTTTCT & TCTCGGTGGCTGGTAGTGA \\
Osterix & CCCTTCTCAAGCACCAATGG & AGGGTGGGTAGTCATTTGCATAG \\
Osteocalcin & CCGGGAGCAGTGTGAGCTTA & AGGCGGTCTTCAAGCCATACT \\
OPG & ATCAGAGCCTCATCACCTT & CTTAGGTCCAACTACAGAGGAAC \\
RANKL & GTGGTCTGCAGCATCGCTC & TCACTCTCCAGAGTCGAGTC \\
\hline
\end{tabular}

\section{Biostatistics}

All values were expressed as means \pm standard deviation. Statistical analyses were performed using SPSS 18.0 software (SPSS Inc, Chicago, IL, USA). Statistical significance was set at $\mathrm{p}<0.05$. Multiple comparisons were used to compare parameters of the tested samples between the different groups and time of observations. Oneway analysis of variance (ANOVA) and Tukey's multiple comparison tests were used to compare the data of various roughness. Mean and standard deviation were calculated.

\section{Results}

\section{SEM shown MC3T3-EI cell morphologic differences on varying roughness of titanium surfaces}

Under SEM, cytoplasmic extensions of MC3T3-E1 cell were more observant on $1 \mu \mathrm{m}$ and $10 \mu \mathrm{m}$ surfaces (Figure 6\&7). Cells would stack up in to a three dimensional structure with more vesicles on the surfaces. On the contrary, machined surface, $0.1 \mu \mathrm{m}$ and $0.01 \mu \mathrm{m}$ surfaces showed less cytoplasmic extensions with more compressed and flat cells, poorer cell adherent properties with chipping noted around the edges (Figures 8-10).

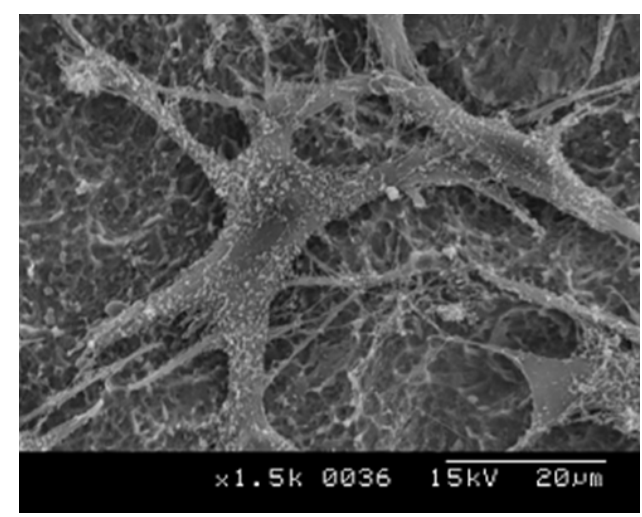

Figure 6 Cell morphology on I $\mu \mathrm{m}$ surface for 72 hours under SEM.

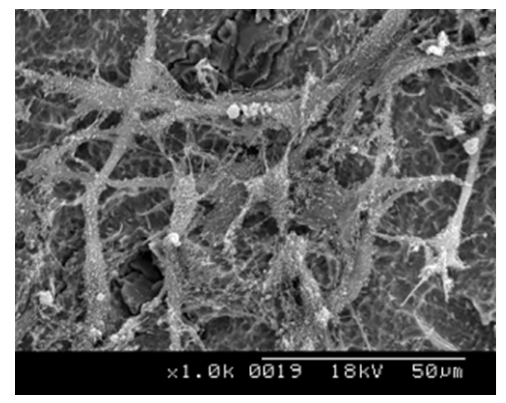

Figure 7 Cell morphology on 10 4 m surface for 72 hours under SEM.

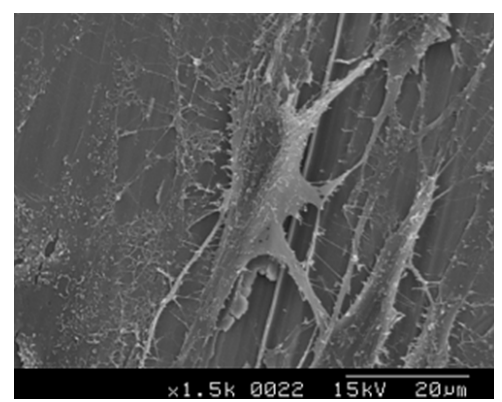

Figure 8 Cell morphology on machined surface for 72 hours under SEM.

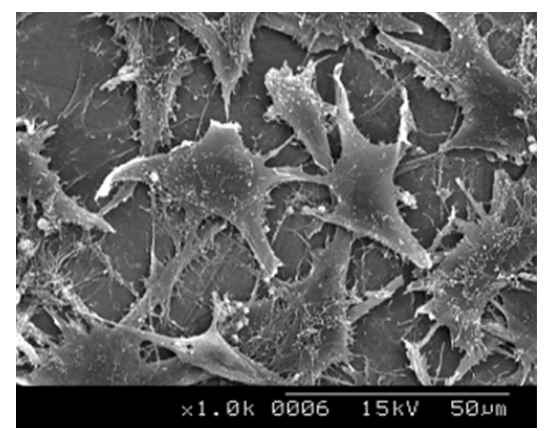

Figure 9 Cell morphology on $0.1 \mu \mathrm{m}$ surface for 72 hours under SEM. 


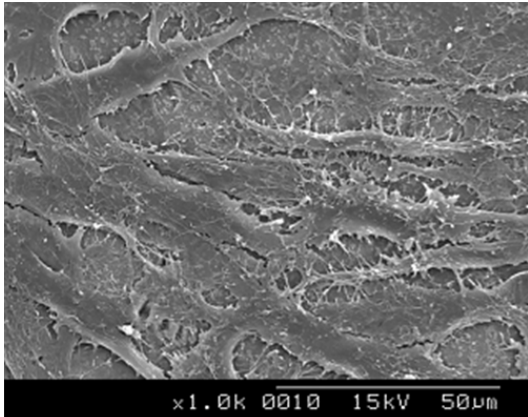

Figure 10 Cell morphology on $0.01 \mu \mathrm{m}$ surface for 72 hours under SEM.

\section{Real time- Q PCR}

After 72 hours, the genetic expression of osteocalcin was most evident on the $1 \mu \mathrm{m}$ surface, demonstrating a structural completed extracellular matrix and that calcification of osteoblasts has initiated (Figure 11). The two indicators of differentiation, Runx-2 and osterix showed delayed expression on the machined surface group, therefore acquiring greater strength than $10 \mu \mathrm{m}, 1 \mu \mathrm{m}$ and $0.1 \mu \mathrm{m}$ roughness surfaces (Figure 12\&13)

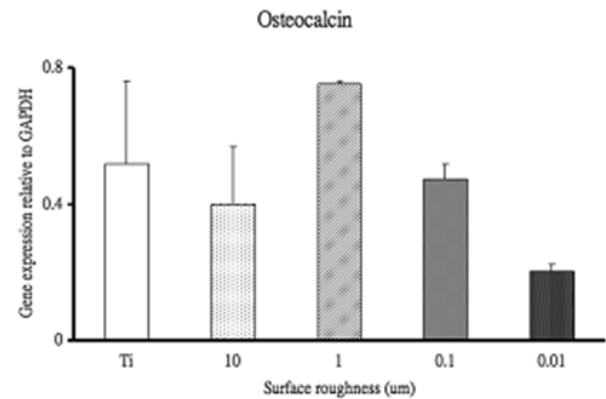

Figure II Osteocalcin gene expressions relative to GAPDH.

Runx-2

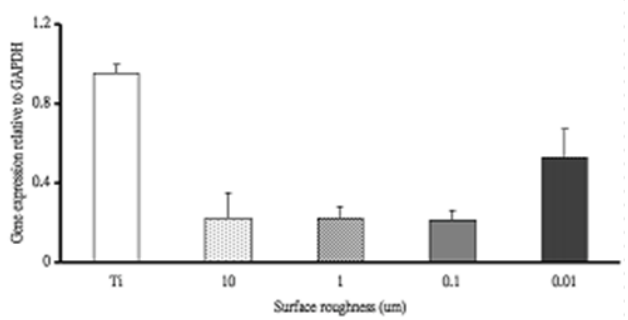

Figure 12 Runx-2 gene expressions relative to GAPDH.

Osterix

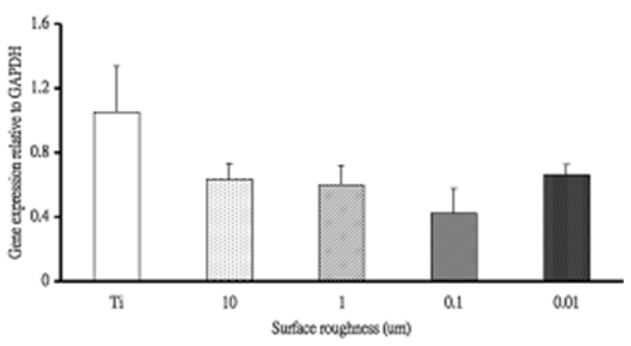

Figure 13 Osterix gene expressions relative to GAPDH.

\section{Discussion}

Early studies of dental implant focus on the materials placed and their biocompatibility. Issues such as persistent chronic inflammation after surgery would cause inability to achieve osteointegration at the connective tissue interface. On the other hand, micromotion of implants is also a potential threat to osteointegration. Surface treatments to add mechanical interlock, such as sintering titanium spherical beads onto the implant surface, has been utilized to enhance primary stability. However, treatments as such would create irregular gaps, leading to mass plaque accumulation once exposed in the oral environment. Peri-implantitis would thus spread apically and severely sabotage the process of osteointegration. With the advancement of sandblasting, acid etching, electrochemistry, and nano technology opens up a wide selection of implant surfaces which has been the focus of recent studies. Composition, surface architecture, roughness and surface energy are four aspects of influence a surface treatment can have on osteoblasts. Yet these four aspects are closely interrelated. ${ }^{19}$ From a biologic point of view, the process of osteointegration undergoes a series of seven steps: protein adsorption, cell adherence, local factor production to promote healing, cell proliferation, cell differentiation, extracellular matrix production, and calcification. ${ }^{20}$ In the above mentioned aspects of implant surface, the most critical factor of osteointegration is surface roughness. Alterations in surface roughness would lead to changes in surface energy, which in turn, generate a different wetting profile and critical surface tension. These changes would affect the protein binding ability of titanium surface and the subsequent cell adhesion. By allowing MC3T3-E1 cell to adhere for the first hour with micromass culture technique, a discrepancy between adherent cells and extracellular matrix would be created due to different titanium surface roughness. It is therefore technically difficult to establish a baseline measurement of cellular growth and other end-products such as collagen I. We further investigate the possibility of surface treatment in altering genetic expression. Prominent gene expression of osteocalcin mRNA observed in surface roughness of $1 \mu \mathrm{m}$ was believed to be related to the integrity of extracellular matrix formation. ${ }^{21}$ Comparing the cellular morphology under SEM and categorizing the surface roughness into rough $(10 \mu \mathrm{m}$, $1 \mu \mathrm{m}$ ) and smooth (machined, $0.1 \mu \mathrm{m}, 0.01 \mu \mathrm{m}$ ), osteoblasts with long processes and polygonal morphology were observed on the rough surfaces, while flat osteoblasts with short processes were seem on smooth surfaces. It is therefore presumed that when osteoblasts form larger collagen fibril bundles, cell growth in layers would be promoted and contact inhibition would be avoided. This is more favorable for osteocalcin mRNA, which is expressed at the late stage in signaling pathway. A surface of $1 \mu \mathrm{m}$ seemed to have a positive influence on osteocalcin mRNA. As to the two genes expressed at the early stage, Runx-2 and Osterix mRNA, both were significantly expressed on smooth surfaces after 72 hours of culturing. Titanium has proven to be a biocompatible material with osteoblasts, and MC3T3-E1 cells were steered towards bone formation. Yet the cellular growth was slower compared with rough surfaces. With surface roughness of $0.1 \mu \mathrm{m}$ and $0.01 \mu \mathrm{m}$, osteoblast differentiation was not significantly enhanced as expected. According to the research by Kilpadi and Lemons, variation in sterile technique and surface treatment may cause differences in critical surface tension. ${ }^{22}$ Consistent surface treatment and sterile techniques were utilized in this experiment, thereby ruling out any possible errors concerning these variables. As Kilpadi and Lemons demonstrated in their study, critical surface tensions of $23 \mu \mathrm{m}$ and $70 \mu \mathrm{m}$ in surface roughness were fairly similar. The authors believed 
that titanium surfaces would form an amorphous titanium oxide layer, reducing the differences created in various surface treatments. In the present study, osteoblast morphology on $0.1 \mu \mathrm{m}$ surface was still dominant by flat cells with short processes. From Martin's study in 1995 using human MG63 osteoblasts, surface binding ability of the $0.2 \mu \mathrm{m}$ surface was significantly lower than the $6-7 \mu \mathrm{m}$ surface. ${ }^{23} \mathrm{We}$ therefore believe that with the formation of an oxide layer, the effect of small size porosity or smooth surface would be less observant.

\section{Conclusion}

This study utilized one single surface treatment procedure to minimize possible bias. By examining the cellular morphology and biochemistry markers, we have found that the surface roughness of $1 \mu \mathrm{m}$ on titanium implant surface possesses most prominent osteogenic factors of the osteoblast cell signaling pathway. An organic matrix structure in morphology would be most suitable for osteoblast adherent and differentiation. ${ }^{24}$

\section{Funding}

None.

\section{Acknowledgments}

We would like to thank Cheng et al. for providing the metal specimens for this study.

\section{Conflicts of interest}

The authors declare no conflict of commercial interests in this submission. This study is self-supported.

\section{References}

1. Klokkevold PR, Nishimura RD, Adachi M, et al. Osseointegration enhanced by chemical etching of the titanium surface. Clin Oral Impl Res. 1997;8(6):442-447.

2. Kasemo B, Lausmaa J. Surface propertities and process of the biomaterialtissue interface. Materials Science and Engineering C1. 1994;115-119.

3. Williams DF. Tissue-material interactions. $J$ Mat Sci. 1987;22(10):3421-3444.

4. Huang W, Yang S, Shao J, et al. Signaling and transcriptional regulation in osteoblast commitment and differentiation. Front Biosci. 2007; 12:3068-3092.

5. Schneider G, Burridge K. Formation of focal adhesions by osteoblasts adhering to different substrata. Exp Cell Res. 1994;214(1):264-269.

6. Stanford CM, Keller JC, Solursh M. Bone cell expression on titanium surfaces is altered by sterilization treatments. J Dent Res. 1994;73(5):1061-1071.

7. Stanford CM, Jacobson PA, Eanes ED, et al. Rapidly forming apatitic mineral in an osteoblastic cell line (UMR 106-01 BSP). J Biol Chem. 1995;270(16):9420-9428.
8. Stanford CM, Brand RA. Toward an understanding of implant occlusion and strain adaptive bone modeling and remodeling. J Prosth Dent. 1999;81(5):553-561.

9. Chihiro M, Galen BS, Rebecca Z, et al. Effects of implant surface microtopography on osteoblast gene expression. Clin Oral Implants Res. 2005;16(6):650-656.

10. Pai H, Lu HK. The relationship between the bone response and surface modification of Ti implants by micro-arc oxidation-a mini-review. Chin Dent J. 2007;26(2):106-113.

11. Yerokhin AL, Nie X, Leyland A, et al. Plasma electrolysis for surface engineering. Surface and Coatings Technology. 1999;122:73-93.

12. Sul YT. The significance of the surface properties of oxidized titanium to the bone response: special emphasis on potential biochemical bonding of oxidized titanium implant. Biomaterials. 2003;24(22):3893-3907.

13. Otto F, Lubbert M, Stock M. Upstream and Downstream Targets of RUNX Proteins. J Cell Bio. 2003;89(1):9-18.

14. Nakashima K, Zhou X, Kunkel G, et al. The novel zinc finger-containing transcription factor Osterix is required for osteoblast differentiation and bone formation. Cell. 2002;08(1):17-29.

15. Ziros PG, Basdra EK, Papavassiliou AG. Runx2: of bone and stretch. Int J Biochem cell Biol. 2008;40(9):1659-1663.

16. Webster TJ, Ejiofor UJ. Increased osteoblast adhesion on nanophase metals: Ti, Ti6Al4V and CoCrMo. Biomaterials. 2004;25(19):4731-4739.

17. Meirelles L, Arvidsson A, Albrektsson T, et al. Increased bone formation to unstable nano rough titanium implants. Clin Oral Impl Res. 2007; 18:326-332.

18. Schwartz Z, Lohmann $\mathrm{CH}$, Sisk $\mathrm{M}$, et al. Local factor production by MG63 osteoblast-like cells in response to surface roughness and $1,25-(\mathrm{OH}) 2 \mathrm{D} 3$ is mediated via protein kinase $\mathrm{C}$ - and protein kinase A-dependent pathways. Biomaterials. 2001;22(7):731-741.

19. Schwartz Z, Kieswetter K, Dean DD, et al. Underlying mechanisms at the bone-surface interface during regeneration. J Periodontal Res. 1997;32(1 pt 2):166-171.

20. Schwartz Z, Lohmann $\mathrm{CH}$, Cochran DL, et al. Bone regulating mechanisms on implant surfaces. Proceedings of the 3rd European Workshop on Periodontology. Germany: Implant Dentistry, Quintessence Publishing; 1999. p. 41-54.

21. Handschel JG, Depprich RA, Kubler NR, et al. Prospects of micromass culture technology in tissue engineering. Head Face Med. 2007;3:4.

22. Kilpadi DV, Lemons JE. Surface energy characterization of unalloyed titanium implants. J Biomed Mater Res. 1994;28(12):1419-1425.

23. Martin JY, Schwartz Z, Hummert TW, et al. Effect of titanium surface roughness on proliferation, differentiation, and protein synthesis of human osteoblast-like cells (MG63). J Biomed Mater Res. 1995;29(3):389-401.

24. Hsin-Chung Cheng, Sheng-Yang Lee, Chih-Mong Tsai, et al. Effect of Hydrogen on Formation of Nanoporous $\mathrm{TiO} 2$ by Anodization with Hydrogen-Fluoride Pretreatment. Electrochemical and Solid-State Letters. 2006;9(11):D25-D29. 